\title{
Quiste de duplicación gástrico en el adulto. A propósito de un caso
}

\author{
Gastric duplication cyst in the adult. A case report
}

$M^{a}$ del Carmen Azorín-Samper*, Isabel Fernández-Palop, Ma Jesús Segura-Jiménez, Cristina Fernández-Martínez, Carlos Flors-Alandi y Félix Checa-Ayet

Servicio de Cirugía General y de Aparato Digestivo, Hospital de Sagunto, Valencia, España

\begin{abstract}
Resumen
El quiste de duplicación gástrico es una anomalía congénita muy rara que representa entre 4 y $9 \%$ de todas las duplicaciones del aparato digestivo. Es una alteración bastante rara en el adulto y la mayoría de los casos se diagnostica en el periodo neonatal. En el adulto suele cursar de forma asintomática y la TC o RMN la descubren de forma incidental, aunque la prueba que la diagnóstica con mayor eficacia es la ecoendoscopia. La extirpación quirúrgica completa de la lesión se considera el tratamiento de elección. Se presenta el caso clínico de una paciente con un quiste de duplicación gástrico diagnosticado de forma incidental.
\end{abstract}

PALABRAS CLAVE: Quiste de duplicación gástrico. Anomalía congénita. Estómago.

\begin{abstract}
Gastric duplication cyst is a very rare congenital anomaly accounting up to 4-9\% of all gastrointestinal tract duplications. It is a quite rare anomaly in adults, the majority of cases are diagnosed in the neonatal period. Gastric duplication cysts are usuaIly asymptomatic in the adult. They are usually discovered incidentally in TAC or RMN, although the best specificity diagnostic test is the echoendoscopy. The best election treatment is the surgical complete extirpation. We describe a case of an adult patient who is diagnosed of the incidentally gastric cyst duplication.
\end{abstract}

KEY WORDS: Gastric duplication cyst. Congenital anomaly. Stomach.

\section{Introducción}

La duplicación del tracto gastrointestinal es una alteración congénita poco frecuente y su incidencia es de 1:4 500 nacimientos. Su localización es variable, pero las más frecuentes son el íleon terminal y el yeyuno $0^{1,2}$.

Los quistes de duplicaciones gástricos (QDG) son muy raros. Representan entre 2 y $9 \%$ de todas las duplicaciones del aparato digestivo ${ }^{3}$. Se presenta el caso clínico de una paciente con un quiste de duplicación gástrico diagnosticado de forma incidental.

\section{Caso clínico}

Mujer de 56 años con antecedentes de hipotiroidismo, remitida al Servicio de Cirugía por el hallazgo radiológico en la TC abdominopélvica de un quiste

\section{Correspondencia:}


abdominal y colelitiasis tras un estudio por infección urinaria de repetición.

La paciente se encontraba clínicamente asintomática y la exploración abdominal no relevó alteraciones de consideración. En las pruebas de laboratorio destacó una elevación de la fosfatasa alcalina y la microglobulina $\beta_{2}$.

En la TC abdominopélvica (Fig. 1) se identificó litiasis biliar y una tumoración quística retroperitoneal, con bordes bien delimitados de $5.7 \times 7.6 \mathrm{~cm}$, y con un contenido homogéneo en su interior. La malformación se encontraba localizada en un plano superior al cuerpo del páncreas, entre bazo, riñón izquierdo y suprarrenal izquierda, y en contacto con la cámara gástrica.

En la RM abdominal (Fig. 2) se confirmó el quiste retroperitoneal situado en la transcavidad de los epiplones. Se hallaba en contacto con la cámara gástrica, bien delimitado y con una pequeña lobulación medial de $7.9 \times 4.7 \mathrm{~cm}$, con un nivel de contenido en su interior.

Se realizó ecoendoscopia en la que se visualizó en la cola de páncreas una lesión quística bien definida de 6.5 $x 6.3 \mathrm{~cm}$, anecoica, con un material hiperecogénico en su interior, de paredes finas y sin septos en su interior. Se observó que la lesión no dependía de la pared gástrica y se identificó un plano de separación con el bazo y el riñón izquierdo. Se practicaron punción y aspiración con aguja fina (PAAF) de la lesión, que obtuvo amilasas de $23380 \mathrm{mU} / \mathrm{ml}$ y CEA de $2198 \mathrm{ng} / \mathrm{ml}$ en el resultado del estudio citológico de la muestra aspirada.

A continuación, la paciente desarrolló una infección de la lesión quística secundaria a la PAAF, por lo que requirió ingreso hospitalario para administración de antibioterapia intravenosa y control evolutivo clínico.

Por último, con la sospecha de QDG, sin poder descartar el probable origen pancreático por la elevada concentración de amilasas y CEA en la PAAF por ecoendoscopia, similar a lo observado en los quistes pancreáticos, se efectuó laparotomía subcostal izquierda. Se identificó un quiste de $7 \times 7 \mathrm{~cm}$ dependiente de la cara posterior gástrica, con una zona de adherencias laxas a la pared gástrica, sin afectación de la mucosa gástrica y sin infiltración de los órganos contiguos. Se realizaron colecistectomía y quistectomía total, incluidas la base del quiste y la zona de implantación gástrica. Se efectuó la apertura de la pieza quirúrgica (Fig. 3) y se reconoció una mucosa similar a la gástrica de aspecto normal y sin lesiones sospechosas de un proceso maligno, por lo que se descartó la posibilidad de ampliar la exéresis gástrica.

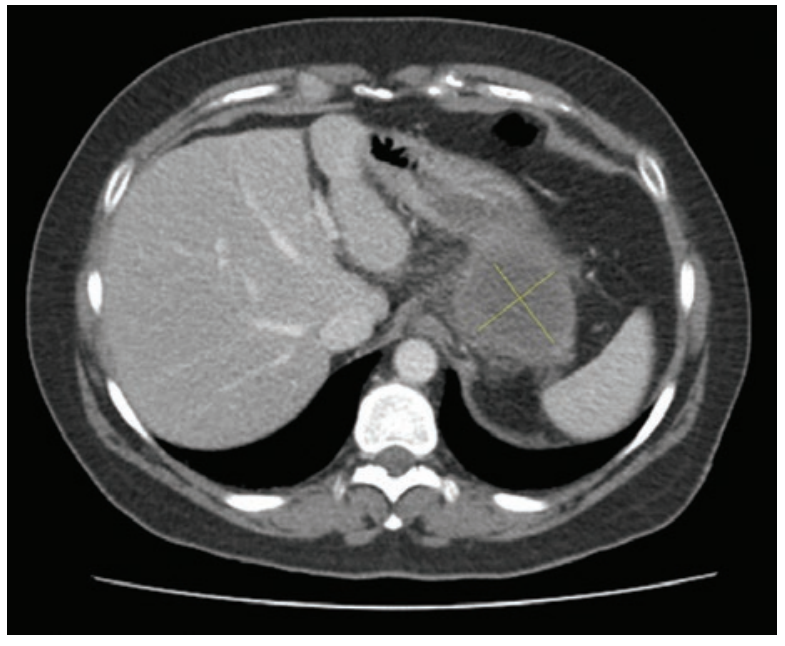

Figura 1. Quiste de duplicación gástrico dependiente de la cara posterior gástrica en la TC abdominopélvica.

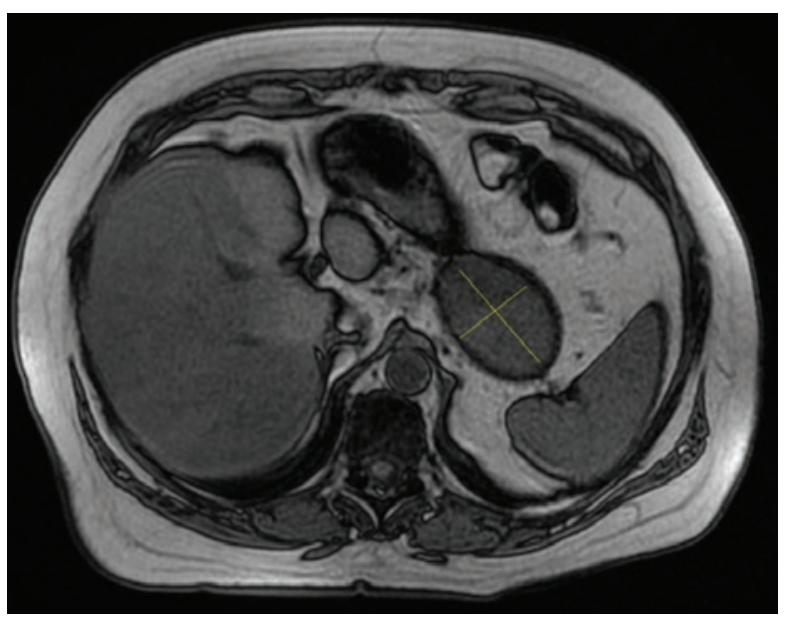

Figura 2. Quiste de duplicación gástrico en contacto con la cara posterior gástrica en la RM abdominal.

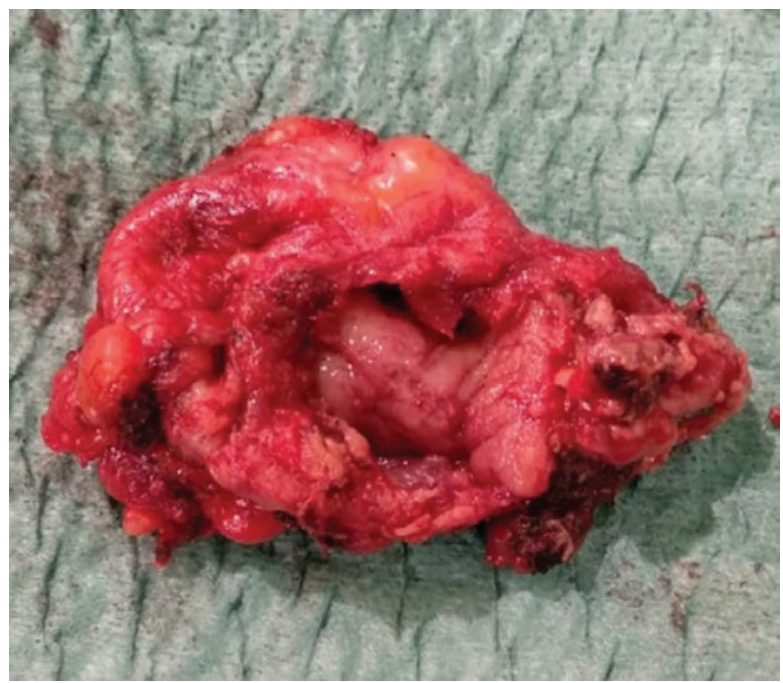

Figura 3. Apertura de la pieza quirúrgica de un quiste de duplicación gástrico. 


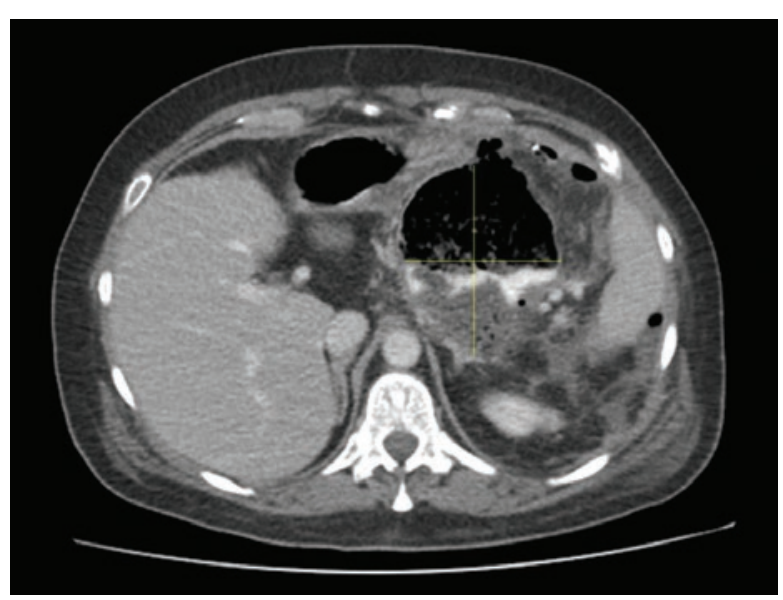

Figura 4. Colección adyacente a la cámara gástrica.

Al tercer día postoperatorio, la paciente presentó malestar general, inestabilidad hemodinámica y en la TC abdominopélvica se observó una colección con gas de $4.3 \times 3.2 \mathrm{~cm}$ adyacente a la cámara gástrica. Se realizó intervención quirúrgica urgente y se reconoció un absceso pútrido espeso con contenido aéreo. Se comprobó la estanquidad de la sutura tras instilación con aire y azul de metileno sin evidenciarse dehiscencia de la sutura.

A los 10 días de la intervención, la paciente presentó un nuevo episodio de empeoramiento clínico y la TC abdominopélvica (Fig. 4) identificó una colección de $7.5 \times 9 \mathrm{~cm}$ que se drenó mediante la colocación de un drenaje percutáneo. Además, se realizó una endoscopia digestiva alta en la que se colocaron grapas en la zona de localización de la sutura gástrica previa, sin evidencia de perforación ni fuga. Tras ello, la paciente presentó una evolución clínica favorable, la colección se resolvió, se inició dieta vía oral progresiva y al final recibió el alta domiciliaria. El resultado anatomopatológico de la pieza confirmó el quiste de duplicación gástrico.

\section{Discusión}

Calder describió en 1733 la primera duplicación del aparato digestivo, mientras que Ladd difundió el término de duplicación en la década de 1930 y Gross realizó las primeras clasificaciones ${ }^{4}$.

Las duplicaciones intestinales se definen por una estructura tubular, quística o diverticular revestida de mucosa gastrointestinal, con una pared de músculo liso y adherida al tracto gastrointestinal, con el que comparte su vascularización y con el que puede existir o no comunicación ${ }^{5,6}$. Estas lesiones deben definirse por su estructura histológica, no por su morfología, y debe establecerse el diagnóstico diferencial con lesiones infecciosas, complicaciones postraumáticas o neoplasias ${ }^{7}$.

La etiopatogenia de la duplicación digestiva se desconoce. Podría tratarse de una perforación anómala del conducto o de la persistencia de un divertículo embrionario. La teoría más aceptada es la formación durante la gastrulación de una fístula entre la cavidad amniótica y el saco vitelino formado en el tejido dorsal de la notocorda entre el ectodermo y el endodermo. También influyen factores medioambientales, como una hipoxia o un traumatismo al inicio de la gestación ${ }^{4,7}$.

La duplicación digestiva en el adulto representa sólo el $5 \%$ de los casos diagnosticados y la gástrica es la menos frecuente (3-4\%) 2 . La localización más común es la curvatura mayor gástrica y predomina en mujeres'. En el $80 \%$ de los casos se manifiesta durante el primer año de vida. En el adulto, lo más frecuente es que curse de modo asintomático y que se descubra de manera incidental; asimismo, la forma asintomática posee el mayor riesgo de transformación neoplásica y el tipo histológico maligno más frecuente es el adenocarcinoma. Además, se han descrito otros tipos como el carcinoma neuroendocrino, el carcinoma de células escamosas y el carcinoma epitelial. No obstante, el QDG puede manifestarse en la forma de dolor abdominal, masa epigástrica, náusea, vómito, pancreatitis recidivantes, HDA, fistulizaciones, oclusiones o suboclusiones ${ }^{3,4,8}$.

La TC y la RM pueden identificar este tipo de lesiones, pero los errores diagnósticos con lesiones sólidas son frecuentes. La mayoría de los autores demuestra que la ecoendoscopia tiene mayor eficacia diagnóstica para estas anomalías y permite además la obtención de material histológico mediante PAAF, fundamental para el diagnóstico diferencial y descartar afección maligna ${ }^{1,2}$.

Existe controversia en el tratamiento de los QDG, sobre todo si son asintomáticos. En estos últimos casos, algunos autores optan por un control evolutivo dado que estas lesiones en el adulto son un hallazgo incidental y el riesgo de transformación maligna es escaso (se han descritos 12 casos en las publicaciones médicas $\left.{ }^{2,3,8-10}\right)$. Sin embargo, no existen datos publicados respecto de la morbimortalidad de una conducta expectante. En los casos sintomáticos, la extirpación quirúrgica completa del quiste se considera el tratamiento de elección para evitar posibles complicaciones, que pueden aparecer en el 20 a $30 \%$ de los casos de QDG con tejido gástrico ectópico en su interior, como obstrucción, torsión, perforación, hemorragia, y por el riesgo de transformación maligna ${ }^{3,7,11}$.

Existen diversas técnicas quirúrgicas descritas, que pueden realizarse mediante abordaje abierto o por vía 
laparoscópica, como la escisión completa del quiste con resección de la pared gástrica circundante, bypass gástrico y gastrectomía parcial o total. En los casos con sospecha de transformación maligna se debe realizar una gastrectomía total con linfadenectomía. La elección de la técnica se basa en ciertas características como el diámetro del quiste, los antecedentes de operaciones abdominales, la posibilidad de transformación maligna y la experiencia quirúrgica del cirujano $0^{3,5}$.

En este caso se optó por realizar quistectomía completa, mediante abordaje abierto, incluidas la base y la zona de implantación gástrica, debido a que no se observaron lesiones sospechosas de malformación maligna.

\section{Financiamiento}

El presente caso clínico no ha recibido ayuda específica de agencias del sector público, sector comercial o entidades sin ánimo de lucro.

\section{Conflicto de intereses}

Los autores declaran no tener conflictos de intereses que puedan afectar el trabajo remitido.

\section{Responsabilidades éticas}

Protección de personas y animales. Los autores declaran que para esta investigación no se han realizado experimentos en seres humanos ni en animales.

Confidencialidad de los datos. Los autores declaran que han seguido los protocolos de su centro de trabajo sobre la publicación de datos de pacientes.

Derecho a la privacidad y consentimiento informado. Los autores han obtenido el consentimiento informado de los pacientes o sujetos referidos en el artículo. Este documento obra en poder del autor de correspondencia.

\section{Bibliografía}

1. García Nebreda M. Quiste de duplicación gástrica de epitelio respiratorio: una lesión infrecuente de difícil diagnóstico diferencial. Cir Esp. 2018.

2. García Pérez B, García Paredes R, Essouri N, Alajarin M, Albaladejo A, Sánchez Torres A, et al. Imagen endoscópica de una duplicacióngástrica. Gastroenterol Hepatol. 2011;34(10):718-9.

3. Tessely H, Montanier A, Chasse E. Gastric duplication cyst with elevated CEA level : a case report. JSCR. 2018:5:1-4.

4. Yandza T, Valayer J. Malformaciones congénitas del estómago. EMC - Pediatría. 2006; 41(1):1-7.

5. Abdalkader M, Al Hassan S, Taha A, Nica I. Complicated gastric duplication cyst in an adult patient: uncommon presentation of an uncommon disease. Radiol Case. 2017;11(8):16-23.

6. Navarrete-Pérez J, Cravioto-Villanueva A, Soria-Céspedes D. Quiste de duplicación gástrica. Informe de un caso. Rev Esp Pat. 2012;45(2):96-9.

7. Herranz Barbero A, Prat Ortells J, Muñoz Fernández M. Duplicación quística intestinal. Caso clínico. Arch Argent Pediatr. 2017;115(4):e233-6.

8. Yamasaki A, Onishi $H$, Yamamoto $H$, lenaga J, Nakafusa Y, Terasaka R, et al. Asymptomatic adenocarcinoma arising from a gastric duplication cyst: a case report. Int J Surg Case Rep. 2016;25:16-20.

9. Seijo Ríos S, Lariño Noia J, Abdulkader Nallib I, Lozano León A, Vieites Pérez-Quintela B, Iglesias García J, et al. Quiste de duplicación gástrico: diagnóstico por punción-aspiración guiada por ecoendoscopia. Rev Esp Enferm Dig. 2008;100(9):586-90.

10. Sethi S, Godhi S, Puri S. Papillary adenocarcinoma in a gastric duplication cyst. Indian J Surg Oncol. 2018;9(1):79-82.

11. Awan A, Tiruneh $F$, Iftikhar $H$, Samuel G. A gastric duplication cyst initially mimicking staghorn calculus. JCPSP. 2018;28(3):S26-7. 